\title{
Editorial: Planning, Conducting and Communicating Systematic Literature Reviews
}

\author{
Mirjana Pejić-Bach ${ }^{2}$ and Narciso Cerpa ${ }^{2}$ \\ ${ }^{1}$ University of Zagreb, Faculty of Economics \& Business, Department of Informatics, Zagreb, Croatia, mpejic@efzg.hr \\ Co-Editor \\ 2 Universidad de Talca, Faculty of Engineering, Curicó, Chile, ncerpa@utalca.cl \\ Editor-in-Chief
}

September 2018

\section{Introduction}

The amount of scientific research conducted and published in peer-reviewed journals is exponentially rising, which creates a problem of information overload. Therefore, the process of tracking the innovative findings in a specific scientific area has become highly complex; simply said, there is a gap between the number of published research papers and amount of time available to study them. A literature review is an attempt of authors to bridge that gap. We could say that literature review is a window that provides the view to the relevant research by providing a summarized insight into scientific development. More precisely, the goals of the literature review are to supply the reader with the following information: '(i) the main achievements in the reviewed field; (ii) the main areas of debate, and (iii) the outstanding research question' [7]. In order to provide this information, the literature review should be conducted in an objective and transparent manner.

There are different definitions for literature review. Firstly, a traditional literature review definition is the one commonly called a theoretical background, which is part of a journal article section that provides the foundations for a study and contextualizes the research questions, providing a central focus. This is the anchor of a research article. Secondly, the literature review that is related to the chapter in a graduate thesis, is usually called a thesis literature review. This is the anchor of an academic thesis or dissertation. Thirdly, the stand-alone literature review articles have the purpose of reviewing a field using and analysing primary studies. In some cases, data from quantitative studies may be combined using meta-analytic techniques [5].

If the stand-alone literature review is conducted in a systematic, formal and rigorous way, it is usually called a Systematic Literature Review (SLR). Systematic literature reviews are very rigorous and have objectives, research questions, and a predefined search strategy [6]. The need for a SLR is born due to the requirement of researchers to summarise all existing findings about a well-defined topic in a thorough, focused, replicable, and unbiased way, with the aim of responding relevant pre-defined questions and drawing well-supported, unbiased and more general conclusions than those obtained from individual research studies [2]. Therefore, SLRs are quite complete and focused, and usually provide important input for a research agenda, with the strict methodology used in order to avoid bias in conducting conclusions based on the prior research analysis. SLR is also considered a prerequisite for performing a meta-analysis [5]. The SLRs were originally used in the field of health to support evidence-based medicine [2], but are nowadays used in virtually every field of science.

In Electronic Commerce, the SLRs is needed to summarize the existing evidence concerning a process, theory, model, or technology. SLR may summarize the empirical evidence regarding the adoption models in electronic commerce, and investigate the gaps in current research with the purpose of suggesting future research, thus providing a framework for developing a research agenda, such as a review on electronic commerce and flexibility [8]. SLRs could also provide a framework or theoretical background for positioning new research activities [3]. Doctoral students may use SLRs for anchoring an academic thesis or dissertation [6], which will summarise, analyse, criticize and build on the literature to encourage and facilitate further academic discussion.

\section{Writing SLR Article}

In order to assure that bias is avoided in the research process, a strict methodological SLR process is used. Hereby we outline the process of writing a SLR paper, mainly based on [11], but also adapted according to other relevant sources, such as [3], [4], [6], [12]. We outline the SLR process in the following four phases that will be briefly discussed:

1. Definition of review scope

2. Conceptualization of topic

3. Literature search

4. Literature analysis and synthesis 


\section{Definition of Review Scope}

Defining the review scope is the most important phase in the SLR process, and it depends on the type of the SLR. Rowe [9] defines three main types of SLRs based on their scope: reviews for describing, for understanding and for explaining, and we refer to them as descriptive, insightful, and elaborative reviews. Descriptive SLRs do not aim to contribute a theory, since their goal is to portray and classify the research findings. Insightful SLRs aim to elaborate on why, how and when something occurs, and focus on the relationship between phenomena using a deductive logic. Elaborative SLRs can be written with the goal to provide insight into some phenomena as a whole, and are based more on broader perspectives, such as conceptual frameworks, descriptive models and theories. Result of such SLR is usually a new research agenda. Based on the goals of the review, its scope should be defined, which is used in the next phase, as the basis for the conceptualization of the topic.

- Examples of descriptive, insightful and elaborative SLRs are [1], [8], [10], respectively.

\section{Conceptualization of Topic}

Based on the review scope, authors should clearly define the key terms, and their definitions, using various sources, such as seminal papers, textbooks, handbooks or encyclopaedias. It is also advisable to use concept maps in order to define the interrelationship between key terms. Concept maps are also a useful way to ensure that all key terms related to the topic are included in the search process.

- For example, [8] used the following key terms: marketplace, electronic procurement, and flexibility; and [10] use the concept map in order to develop a research agenda, as presented in Figure 1 in their paper.

\section{Literature Search}

Based on the conceptualization of the topic, the literature search phase is defined. This search strategy must be documented with the purpose of communicating the rigor and replicability of the process to the reader. In this phase, keywords are usually defined in a more precise manner, compared to the previous phase.

- For example, [8] report that they use specific combinations of keywords selected in the conceptualization phase: (i) (Marketplace AND Electronic Procurement AND Flexibility); (ii) (Marketplace AND E-Procurement AND Flexibility), (iii) (E-Marketplace AND E-procurement AND Flexibility), and (iv) (Flexibility AND Eprocurement Marketplace).

Another important method is the application of inclusion, exclusion and quality criteria to evaluate each potential primary study. If a primary study does not have the expected quality level, it is excluded. Preferred Reporting Items for Systematic Reviews and Meta-Analyses (PRISMA) flow diagram is often used as a method for systematic planning and representation of literature research [5]. It includes a careful tracking of the following items: \# of records identified through database searching; \# of additional records identified through other sources; \# of records after duplicates removed; \# of records screened; \# of records excluded; \# of full-articles assessed for eligibility; \# of full articles excluded with reasons; \# of studies included in qualitative analysis; and \# of studies included in quantitative analysis.

In this phase, the decision on the literature sources should also be made. The usual choice are papers published in peer-reviewed journals indexed in Scopus and/or Web of Science. However, the change is rapid in the area of Electronic Commerce; it is likely that the inclusion of the papers published in scientific conference proceedings is also needed. In that case, some standard of quality for the scientific conferences should be used, such as indexing in Scopus or ISI Proceedings databases. It is also possible to include backward and forward citations, as a source of relevant papers [4].

- $\quad$ For example, [8] report that they searched the following sources: IEEE Xplore, ACM Digital Library, Citeseer Library, Science Direct, and ISI Web of Science, and present a flow diagram of their search process (Figure 6 in their paper).

\section{Literature Analysis and Synthesis}

Collected search results are used for the literature analysis and synthesis. Literature analysis can be conducted based on author-centric and concept-centric approach, while the second is more often used. In order to facilitate this process, [12] propose the usage of the concept matrix, which is used as the basis for the analysis and synthesis of papers.

- For example, [10] develop a concept matrix (Table 1 in their papers) based on a concept map (Figure 1 in their paper). 
In the case of quantitative studies, the data may be combined using meta-analysis techniques, increasing the probability of finding real effects that smaller individual studies cannot find [3]. However, one has to be careful because the increased power of meta-analysis may detect small bias as well as true effects.

Possible results of the literature synthesis are defined by [7]: (i) the major achievements in the reviewed field, (ii) the main area of debate, and (iii) the outstanding research question, but which should be delivered taking into account the type of SLR. Descriptive SLR may conduct only basic bibliometric analysis, portraying the main authors, journals, and topics, based on already developed research agenda. Insightful SLR may conduct the various analysis of relationships between various phenomena. Elaborative $S L R$ should aim at developing a new research agenda, or refine an existing one. Research agenda is even defined by [9] as an obligatory part of every SLR, but this is not applicable for descriptive and insightful reviews.

Properly conducted SLR should diminish the probability of a bias in the review process; publication bias (if any) from the primary studies remains in the SLR [3], and conclusions should be made with considering those. For example, when the research results are consistent across the studies, it is likely that a robust phenomenon has been examined. On the other hand, if the results are inconsistent, the sources of variation may need to be studied [3].

\section{Summary}

The aim of this editorial is to discuss and provide a methodological approach to plan, conduct, and communicate literature reviews in a systematic, formal and rigorous way. For achieving our purpose, we summarize the relevant literature on this topic.

A systematic literature review begins by defining a review protocol that specifies the research questions to be answered and the systematic methods that will be used to undertake the review - for example, the defined search strategy that will help to detect all the relevant literature as required. We outline the SLN methodology in four phases, but which are not mutually exclusive, and are of iterative nature. For example, the conceptualization of the topic can be adjusted in the process of the literature search.

In order to support prospective authors, various papers provide practical advice for writing SLRs. For example, [7] provides the following practical advice on literature search: '(i) keep track of the search items you use; (ii) keep track of papers that you cannot access immediately; (iii) use a paper management system; (iv) define early in the process clear criteria for exclusion, (v) do not just look for research papers, but also seek previous reviews'. In other words, authors should plan their work ahead as much as possible, and make sure that they keep track of every step of SLR methodology.

Detailed guidance and examples on writing specific parts of the SLRs are provided by [5], such as the rationale for conducting a literature review on the specific topic. Finally, the prospective authors should be aware that due to the elaborate work process, SLRs require substantially more effort than traditional literature reviews [2], [3]. On the other hand, this process is in many instances rewarding, since properly executed SNLs provide a solid platform for developing a new research, and are important for the development of rapid growing fields of science, such as Electronic Commerce.

\section{References}

[1] F. Karimi-Alagheband, S. Rivard, S. Wu, and S. Goyette. An assessment of the use of transaction cost theory in information technology outsourcing, Journal of Strategic Information Systems, vol. 20, no. 2, pp. 125-138, 2011.

[2] K.S. Khan, G. Ter Riet, J. Glanville, A.J. Sowden, and J. Kleijnen, Undertaking systematic review of research on effectiveness, CRD's guidance for those carrying out or commissioning reviews, NHS Centre for Reviews and Dissemination, University of York, U.K., CRD Report Number 4, second ed., 2001.

[3] B.A. Kitchenham, Procedures for undertaking systematic reviews, Computer Science Department, Keele University (TR/SE0401) and National ICT Australia Ltd. ( 0400011T.1), Joint Technical Report, 2004.

[4] Y. Levy and T. J. Ellis, A systems approach to conduct an effective literature review in support of information systems research, Informing Science Institute, vol. 9, pp. 181-212, 2006.

[5] D. Moher, A. Liberati, J. Tetzlaff, and D.G. Altman, Preferred reporting items for systematic reviews and metaanalyses: The PRISMA statement, PLoS Medicine, vol. 6, no. 7: e1000097, 2009

[6] C. Okoli and K. Schabram, A guide to conducting a systematic literature review of information systems research, Sprouts: Working Papers in Information Systems, vol. 10, no. 26, pp. 1-46, 2010.

[7] M. Pautasso, Ten simple rules for writing a literature review, PLoS Computational Biology, vol. 9, no. 7, e1003149, 2013

[8] S. Robaty Shirzad and D. Bell, A systematic literature review of flexible e-procurement marketplace, Journal of Theoretical and Applied Electronic Commerce Research, vol. 8, no. 2, pp. 49-70, 2013.

[9] F. Rowe, What a literature review is not: Diversity, boundaries and recommendations, European Journal of Information Systems, vol. 23, no. 3, pp. 240-250, 2014. 
[10] D. Suša Vugec, K. Tomičić-Pupek and V. B. Vukšić, Social business process management in practice: Overcoming the limitations of the traditional business process management, International Journal of Engineering Business Management, vol. 10, 2018.

[11] J. vom Brocke, A. Simons, B. Niehaves, B. Niehaves, K. Reimer, R. Plattfaut, and A. Cleven, Reconstructing the giant: on the importance of rigour in documenting the literature search process, in Proceedings $17^{\text {th }}$ European Conference on Information Systems (ECIS), Verona, 2009, pp. 2206-2217.

[12] J. Webster and R. T. Watson. Analyzing the past to prepare for the future: Writing a literature review, MIS Quarterly, vol. 26, no. 2, pp. xiii-xxiii, 2002. 